\title{
LHC data challenges the contemporary parton-to-hadron fragmentation functions
}

\author{
David d'Enterria \\ CERN, PH Department, CH-1211 Geneva 23, Switzerland \\ E-mail: dde@cern.ch
}

\section{Kari J. Eskola}

Department of Physics, University of Jyväskylä, P.O. Box 35, FI-40014 University of Jyväskylä, Finland

Helsinki Institute of Physics, University of Helsinki, P.O. Box 64, FI-00014, Finland

E-mail: kari.eskola@jyu.fi

\section{Ilkka Helenius}

Department of Physics, University of Jyväskylä, P.O. Box 35,

FI-40014 University of Jyväskylä, Finland

Helsinki Institute of Physics, University of Helsinki, P.O. Box 64, FI-00014, Finland

E-mail: ilkka.helenius@jyu.fi

\section{Hannu Paukkunen*}

Department of Physics, University of Jyväskylä, P.O. Box 35,

FI-40014 University of Jyväskylä, Finland

Helsinki Institute of Physics, University of Helsinki, P.O. Box 64, FI-00014, Finland

E-mail: hannu.paukkunenejyu.fi

\begin{abstract}
We discuss the inclusive high-pT charged-particle production in proton-proton collisions at the LHC. The experimental data are compared to the NLO perturbative QCD calculations employing various sets of parton-to-hadron fragmentation functions. Most of the theoretical predictions are found to disastrously overpredict the measured cross sections, even if the scale variations and PDF errors are accounted for. The problem appears to arise from the presently too hard gluon-tohadron fragmentation functions.
\end{abstract}

XXII. International Workshop on Deep-Inelastic Scattering and Related Subjects, 28 April - 2 May 2014

Warsaw, Poland

${ }^{*}$ Speaker. 


\section{Motivation}

The principal motivation for our study [1] was the observation that the next-to-leading order (NLO) perturbative QCD (pQCD) calculations for inclusive charged hadron production shown along with the published $p+p$ data from CMS [2] and ALICE [3] appeared to clearly overshoot the measurements at large transverse momentum $\left(p_{T}\right)$. This called for a systematic study to chart the different sources of theory uncertainties and thereby, hopefully, identify the cause of the apparent mismatch.

\section{Framework}

The inclusive high- $p_{T}$ charged-particle $\left(h_{3}\right)$ production in collision of two hadrons $h_{1}$ and $h_{2}$ can be computed as a convolution of the initial-state parton distribution functions $f_{i}\left(x, \mu_{\text {fact }}^{2}\right)$ (PDFs), final-state parton-to-hadron fragmentation functions $D_{k \rightarrow h_{3}}\left(z, \mu_{\text {frag }}^{2}\right)$ (FFs), and partonic coefficient functions $d \sigma^{i+j \rightarrow k+X}\left(\mu_{\text {ren }}^{2}, \mu_{\text {fact }}^{2}, \mu_{\text {frag }}^{2}\right)$ as

$$
d \sigma\left(h_{1}+h_{2} \rightarrow h_{3}+X\right)=\sum_{i j k} f_{i}^{h_{1}} \otimes d \sigma^{i+j \rightarrow k+X} \otimes f_{j}^{h_{2}} \otimes D_{k \rightarrow h_{3}},
$$

where $\mu_{\text {ren }}^{2}, \mu_{\text {fact }}^{2}$, and $\mu_{\text {frag }}^{2}$ denote the renormalization, factorization, and fragmentation scales, respectively. Figure 1 presents a schematic illustration of the process ingredients. In practice, we evaluate the cross sections to NLO in strong coupling $\alpha_{s}\left(\mu_{\text {ren }}^{2}\right)$ utilizing the public INCNLO code $[4,5]$. For the PDFs we use CT10NLO [6] and its error sets. The FF uncertainty is estimated by performing the calculations with various parametrizations: Kretzer (KRE) [7], KKP [8], BFGW [9], HKNS [10], AKK05 [11] DSS [12, 13], and AKK08 [14]. From all these, only HKNS offers a possibility to estimate the propaga-

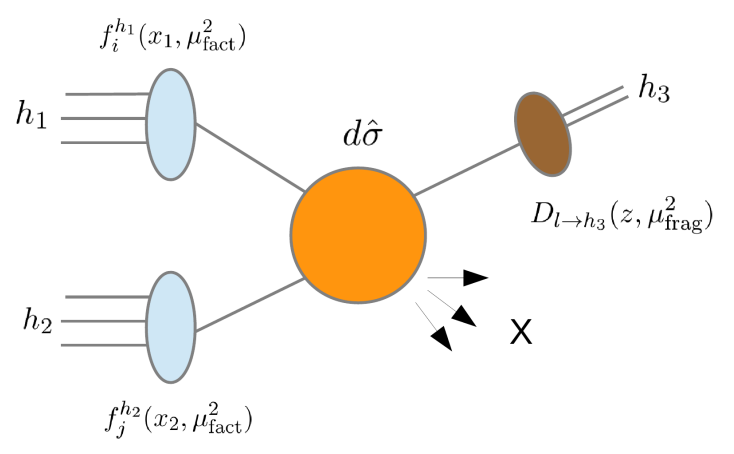

Figure 1: Ingredients of inclusive hadron production in hadronic collisions.

tion of FF uncertainties to further observables. We illustrate the differences in these FFs by plotting, in Figure 2, the FFs for $u$-quarks and gluons. While the $u$-quark FFs are all rather tightly packed together, the spread among the different gluon FFs is huge. The main reason for the large differences is that the $e^{+} e^{-}$annihilation data which constitutes the bulk of the FFs constraints are predominantly sensitive to the quark fragmentation, leaving the gluons rather unconstrained. To improve the situation DSS and AKK08 included also hadroproduction data from RHIC, SPS and Tevatron, but predominantly at rather low values of $p_{T}$ where the NLO pQCD calculations are in doubt (see later). Along with the LHC $\mathrm{p}+\mathrm{p}$ runs at several center-of-mass energies it has now become possible to fit the FFs at higher $p_{T}$ and also cross-check between different experiments. As illustrated in Figure 3, the gluon fragmentation dominates the cross sections up to the highest measurable values of $p_{T}$ and the large spread of gluon FFs seen in Figure 2 should therefore make a difference at the LHC. 

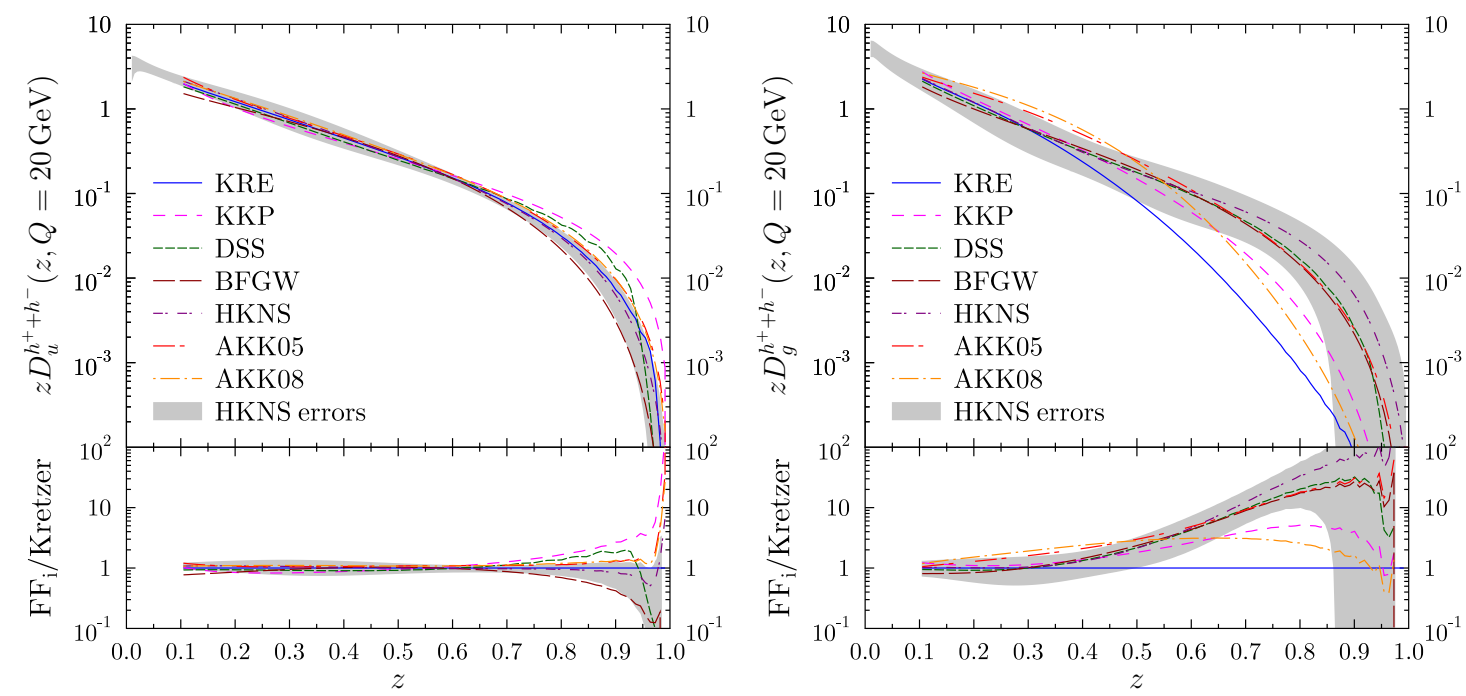

Figure 2: The absolute charged-hadron FFs for $u$-quarks (left) and gluons (right) at $\mu_{\text {frag }}=20 \mathrm{GeV}$, and their ratios to Kretzer FFs. Figure from [1].

Our default choice for the involved QCD scales is $\mu_{\text {ren }}=$ $\mu_{\text {fact }}=\mu_{\text {frag }}=p_{T}$, and we explore the sensitivity of the NLO calculation to this particular choice by varying the scales independently by a factor of two. However, we exclude the variations with

$$
\frac{\mu_{\text {ren }}}{\mu_{\text {frag,fact }}}=4 \text { or } \frac{1}{4},
$$

as this gives rise to artificially

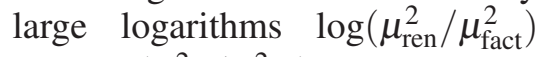
and $\log \left(\mu_{\text {ren }}^{2} / \mu_{\text {frag }}^{2}\right)$, in terms involving partonic splitting functions $P_{i j}$. Schematically,

$$
\frac{\alpha_{s}\left(\mu_{\text {ren }}^{2}\right)}{2 \pi} \log \left(\frac{\hat{s}}{\mu_{\text {fact }, \text { frag }}^{2}}\right) P_{i j} \approx \frac{\alpha_{s}\left(\mu_{\text {fact,frag }}^{2}\right)}{2 \pi} \log \left(\frac{\hat{s}}{\mu_{\text {fact }, \text { frag }}^{2}}\right) P_{i j}+\frac{\alpha_{s}^{2}\left(\mu_{\text {ren }}^{2}\right)}{2 \pi} \frac{\beta_{0}}{4 \pi} \log \left(\frac{\mu_{\text {fact,frag }}^{2}}{\mu_{\text {ren }}^{2}}\right) \log \left(\frac{\hat{s}}{\mu_{\text {fact,frag }}^{2}}\right) P_{i j},
$$

which follows from the QCD renormalization group equation $\left(\beta_{0}\right.$ is the first term in the QCD $\beta$-function and $\hat{s}$ denotes the partonic center-of-mass energy). Whereas the first term tends to moderate effect of varying $\mu_{\text {fact,frag }}$, the second term can become large. 


\section{Results}

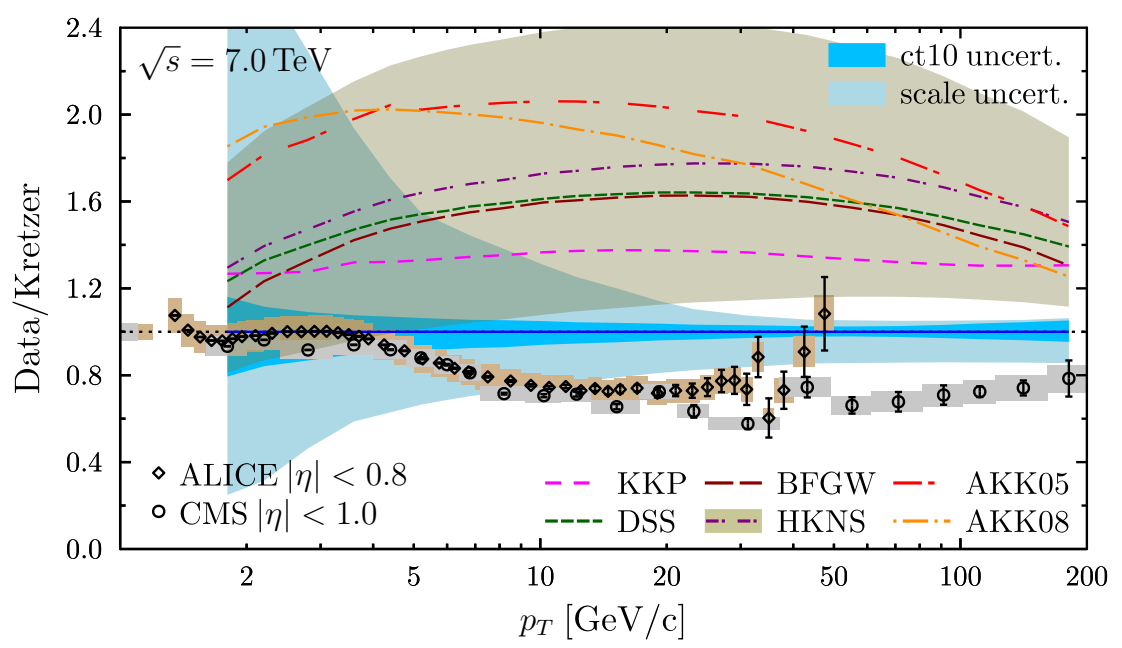

Figure 4: Comparison between the CMS and ALICE $7 \mathrm{TeV}$ data and the calculations with various FFs. Figure adapted from [1].

Figure 4 shows a representative example of what we find when contrasting the NLO calculations against the measurements. The data as well as all the calculations with various FFs have been normalized by the results obtained with the Kretzer FFs. The main points we want to emphasize are:

- The CMS and ALICE data are in a fair agreement.

- The PDF-originating uncertainty (dark blue band) turns out rather small, around 5\% at high $p_{T}$.

- The scale uncertainty (light blue band) is huge at small $p_{T}$, but levels off beyond $p_{T} \sim$ $10 \mathrm{GeV}$.

- The calculation with Kretzer FFs gives the best description of the data, being around $20 \%$ above the data beyond $p_{T} \sim 10 \mathrm{GeV}$. The data-to-theory ratio is remarkably flat despite the fact that the absolute cross section drops around eleven orders of magnitude.

- All other FFs overshoot the data even more. The error band of HKNS is large but not large enough to enclose the data.

Although we presented here only the $7 \mathrm{TeV}$ LHC data as an example, the situation remains more or less the same when considering the other LHC energies and also with the data from the CDF collaboration [15] (see Ref. [1] for more details). It should be noted that related processes like inclusive jet $[16,17]$ or isolated photon production $[18,19]$ at the LHC are in agreement with the NLO calculations. This reinforces the idea that the mismatch between the LHC data and the NLO calculations is indeed due to the current gluon FFs which have either (almost) no data constraints or have been constrained at very low $p_{T}$ where the scale uncertainty is enormous. The scale uncertainty is, however, not the only reason why the region below $p_{T} \sim 10 \mathrm{GeV}$ should be discarded 
from charged-hadron FF fits: The qualitative difference between the $\left(K^{+}+K^{-}\right) /\left(\pi^{+}+\pi^{-}\right)$and $\left(p^{+}+p^{-}\right) /\left(\pi^{+}+\pi^{-}\right)$ratios measured by ALICE $[20,21]$ indicate that the baryon production at this low- $p_{T}$ region cannot be considered being just independent parton-to-hadron fragmentation but different (collective?) physics seems to be involved. From $p_{T} \sim 10 \mathrm{GeV}$ onwards such differences appear to disappear and the NLO calculations, like the ones presented here, should be adequate.

Despite the significant data-to-theory mismatch in the case of absolute cross sections it has been noticed [3] that the ratios of cross section between different $\sqrt{s}$ are, however, much better described by the NLO pQCD. This is demonstrated in Figure 5 presenting some ALICE data and calculations with two different FFs, Kretzer and DSS. Indeed, even DSS which grossly overshoots the absolute spectra (see Figure 4) is, more or less, consistent with the data. This follows from the fact that the cross-section ratios in Figure 5 are more sensitive to the shape of the gluon FFs and not that much to their absolute magnitude.
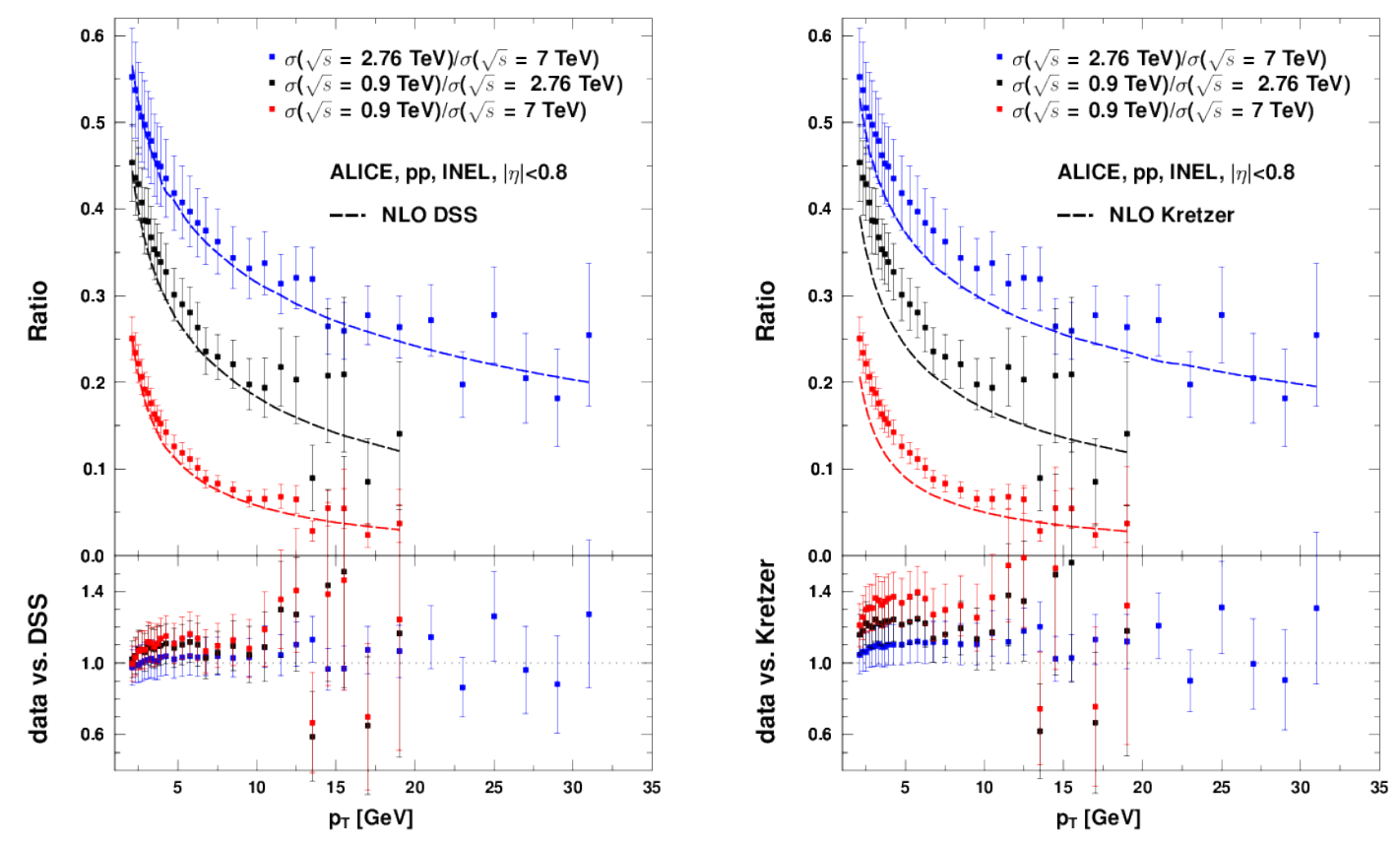

Figure 5: Ratios between the charged-hadron yields at different center-of-mass energies. The data points are from ALICE [3] (constructed by dividing the cross sections and adding the uncertainties in quadrature) and the calculations (dashed lines) are obtained using the DSS (left) and Kretzer (right) FFs.

\section{Summary}

In conclusion, we have found that none of the current sets of FFs can optimally describe the LHC (or Tevatron) data for inclusive charged hadron production at $p_{T} \gtrsim 10 \mathrm{GeV}$. Below $p_{T} \sim$ $10 \mathrm{GeV}$ the scale uncertainty is enormous prohibiting to make practically any conclusion and, in addition, in this low- $p_{T}$ region there are evidence for excess baryons which do not seem to originate from independent parton-to-hadron fragmentation. For these reasons we conclude that only the region $p_{T} \gtrsim 10 \mathrm{GeV}$ should be used in the forthcoming fits of charged-hadron FFs. 


\section{Acknowledgments}

We acknowledge the financial support from the Magnus Ehrnrooth Foundation (I.H.) and from the Academy of Finland, Project No. 133005.

\section{References}

[1] D. d'Enterria, K. J. Eskola, I. Helenius and H. Paukkunen, Nucl. Phys. B 883 (2014) 615 [arXiv:1311.1415 [hep-ph]].

[2] S. Chatrchyan et al. [CMS Collaboration], Eur. Phys. J. C 72 (2012) 1945 [arXiv:1202.2554 [nucl-ex]].

[3] B. B. Abelev et al. [ALICE Collaboration], Eur. Phys. J. C 73 (2013) 2662 [arXiv:1307.1093 [nucl-ex]].

[4] http://lapth.cnrs.fr/PHOX_FAMILY/readme_inc.html

[5] F. Aversa, P. Chiappetta, M. Greco and J. P. Guillet, Nucl. Phys. B 327 (1989) 105.

[6] H. -L. Lai, M. Guzzi, J. Huston, Z. Li, P. M. Nadolsky, J. Pumplin and C. -P. Yuan, Phys. Rev. D 82 (2010) 074024 [arXiv:1007.2241 [hep-ph]].

[7] S. Kretzer, Phys. Rev. D 62 (2000) 054001 [hep-ph/0003177].

[8] B. A. Kniehl, G. Kramer and B. Potter, Nucl. Phys. B 582 (2000) 514 [hep-ph/0010289].

[9] L. Bourhis, M. Fontannaz, J. P. Guillet and M. Werlen, Eur. Phys. J. C 19 (2001) 89 [hep-ph/0009101].

[10] M. Hirai, S. Kumano, T. -H. Nagai and K. Sudoh, Phys. Rev. D 75 (2007) 094009 [hep-ph/0702250].

[11] S. Albino, B. A. Kniehl and G. Kramer, Nucl. Phys. B 725 (2005) 181 [hep-ph/0502188].

[12] D. de Florian, R. Sassot and M. Stratmann, Phys. Rev. D 75 (2007) 114010 [hep-ph/0703242].

[13] D. de Florian, R. Sassot and M. Stratmann, Phys. Rev. D 76 (2007) 074033 [arXiv:0707.1506 [hep-ph]].

[14] S. Albino, B. A. Kniehl and G. Kramer, Nucl. Phys. B 803 (2008) 42 [arXiv:0803.2768 [hep-ph]].

[15] T. Aaltonen et al. [CDF Collaboration], Phys. Rev. D 79 (2009) 112005 [Erratum-ibid. D 82 (2010) 119903] [arXiv:0904.1098 [hep-ex]].

[16] S. Chatrchyan et al. [CMS Collaboration], Phys. Rev. D 87 (2013) 11, 112002 [arXiv:1212.6660 [hep-ex]].

[17] G. Aad et al. [ATLAS Collaboration], Phys. Rev. D 86 (2012) 014022 [arXiv:1112.6297 [hep-ex]].

[18] S. Chatrchyan et al. [CMS Collaboration], Phys. Rev. D 84 (2011) 052011 [arXiv:1108.2044 [hep-ex]].

[19] G. Aad et al. [ATLAS Collaboration], Phys. Lett. B 706 (2011) 150 [arXiv:1108.0253 [hep-ex]].

[20] B. B. Abelev et al. [ALICE Collaboration], arXiv:1401.1250 [nucl-ex].

[21] R. Preghenella [ALICE Collaboration], arXiv:1304.0899 [hep-ex]. 